\title{
A 4-year-old child with fever and persistent cough
}

\section{Case report}

The presenting patient was a 4-year-old female who was hospitalised with fever and cough, which had persisted for the previous 2 months. There had been no prior respiratory symptomatology and the perinatal course had been uncomplicated. The patient was subsequently referred to the Dept of Pulmonary Medicine (Federal University of Rio de Janeiro, Rio de Janeiro, Brazil) after abnormal chest radiography (figure 1).

An echocardiogram demonstrated normal cardiac anatomy, although pericardial effusion was noted. On pericardicentesis, a turbid and haemorrhagic liquid was revealed, which had negative cultures for a number of common microorganisms. Pericardium biopsy proved inconclusive.

At rest, the patient's respiratory rate was 20 breaths per minute, with an absence of dyspnoea or cyanosis. Bilateral basal rales were observed on auscultation. The systemic examination was otherwise normal.

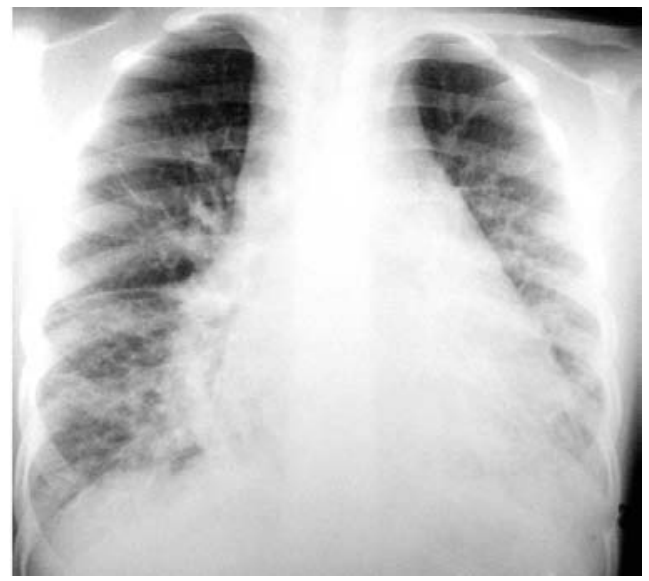

Figure 1

Chest radiograph.

Task 1

Interpret the chest radiograph.
C.C. Sant'Anna ${ }^{1}$

A.L.A.D. Nogarol ${ }^{1}$

M. de Fátima ${ }^{1}$

G.M. Leal ${ }^{2}$

K. Madi ${ }^{3}$

L. Alves ${ }^{1}$

Depts of ${ }^{1}$ Pulmonary Medicine and ${ }^{3}$ Pathology, Instituto de Puericultura e Pediatria Martagão Gesteira, Federal University of Rio de Janeiro, and

${ }^{2}$ Dept of Thoracic Surgery, Hospital da Lagoa, Rio de Janeiro, Brazil.

\section{Correspondence:}

A.L.A.D. Nogarol Division of Paediatric Pulmonology Dept of Paediatrics

Brigadeiro Trompowski Avenue ( $s / n$ ) Instituto de Puericultura e Pediatria Martagão Gesteira, UFRJ

Rio de Janeiro

Brazil

E-mail:alinediv@terra.com.br 


\section{Answer 1}

The chest radiograph revealed diffuse interstitial infiltrates, Kerley B lines and an enlarged cardiac shadow.

The patient was treated with antibiotics for common microorganisms, and diuretics. The pericardial effusion and cough improved, but the pulmonary infiltrates persisted.

The results of complementary examinations were as follows: negative reaction to tuberculin skin test; and sputum smear negative for acid-fast bacillus. The chest computed tomography (CT) scan that was taken at this time is shown in figure 2 .

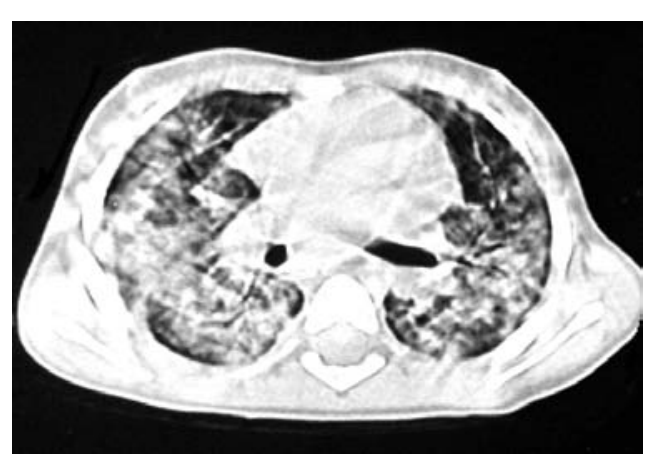

Figure 2

Chest CT scan.

\section{Task 2}

Interpret the CT scan.

\section{Answer 2}

The CT scan showed parenchymal inhomogeneity with increased interstitial markings.

Due to the persistence of fever and pulmonary infiltrates, treatment for tuberculosis (TB), with rifampicin, isoniazid and pyrazinamide, was initiated empirically with fever improvement but no radiological improvement. After 5 months, chest radiography continued to show diffuse interstitial infiltrates, Kerley B lines and enlarged cardiac shadow. An echocardiogram demonstrated pericardial thickening and normal cardiac anatomy. Helical CT showed no vascular anomalies.

\section{Task 3}

At this point, what would you do? 


\section{Answer 3}

An open-lung biopsy was performed at this time.

Following the open-lung biopsy, histological examination showed extreme dilatation of subpleural lymph vessels, compatible with congenital pulmonary lymphangiectasis (CPL; figure 3 ).

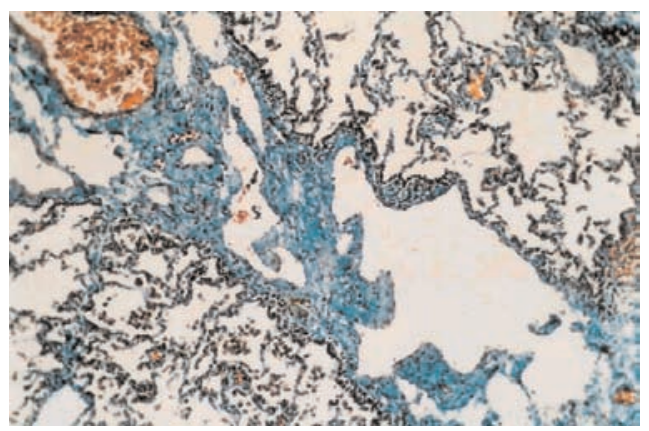

Figure 3

Histopathological findings from the open-lung biopsy material.

\section{Clinical course}

One month later, left-sided pleural effusion was noted and the thoracentesis showed an opalescent liquid of pH 7.85, triglycerides $1,800 \mathrm{mg}$ per $\mathrm{dL}$, cholesterol $93 \%$, and a cell count with a predominance of lymphocytes.

\section{Task 4 \\ What kind of pleural effusion can be observed?}

\section{Answer 4}

The pleural effusion was shown to be chylothorax, by the fat content and the predominance of lymphocytes in the opalescent pleural effusion.

Parenteral nutrition with lipids was initiated with a decrease in pleural effusion, but it subsequently re-accumulated and the patient underwent three more thoracocenteses. Over the following 5 months, the patient was hospitalised on three occasions for respiratory distress due to recurrent chylothoraces. A thoracotomy revealed extreme dilatation of the lymphatic channels over the parietal pleura and a pleurodesis with $2 \%$ silver nitrate solution was performed.

The child was recommended a low-fat diet and remained asymptomatic for 3 years. After this uneventful period, the patient presented with body bruising. Her blood tests showed 109,000 platelets, activated partial thromboplastin time 1.37 (normal value $<1.3$ ), prothrombin time 1.27 (normal value $<1.20$ ), fibrinogen 103 (normal range 200-400), and bleeding time, D-dimers, factors assays (VIII, IX and XI) and von Willebrand studies were normal. After 3 months, the patient died aged 7 years due a massive haemoptysis in another hospital.

\section{Discussion}

Pulmonary lymphangiectasis is a rare condition characterised by dilatation of the pulmonary lymphatics [1]. It may be of two types: 1) CPL, probably by anomalous development of pulmonary lymphatics; and 2) acquired, secondary to obstruction of lymphatic drainage or to congenital cardiopathy with obstruction of venous return. The commonest form of CPL involves a premature onset, with respiratory discomfort in the neonatal period, which is generally fatal $[2,3]$.

Noonan et al. [4] divided CPL into three groups as follows: 1) as part of generalised lymphangiectasis; 2) secondary to pulmonary venous obstruction; and 3) isolated. In the diffuse form of isolated CPL, the disease may involve all areas of the lungs, whereas in the localised form the lungs are only partially affected. The diffuse form is more frequent in newborns, with premature onset, and is often fatal. The patient who presented here matched the diagnosis of diffuse isolated $\mathrm{CPL}$, since no other anomaly was presented and both lungs were affected in a diffuse manner. However, there was no premature onset and she had been asymptomatic until 4 years of age.

Three cases of late-onset diffuse isolated CPL that have been reported in the literature presented 
similar features, including coughing, diffuse reticulonodular infiltrate, pericardial effusion and chylothorax [5-7], although there was no evidence of cardiopathy or lymphatic obstruction. In the current case, the turbid and haemorrhagic pericardium effusion may be re-interpreted as chylum, with blood and apoptotic lymphocytes appearing as neutrophils.

Diagnosis is based upon lung biopsy and histological examinations. Lymphography is useful to exclude lymphatic obstruction, but, in the current authors' hospital, this was difficult to perform. High-resolution CT (HRCT) is useful for defining parenchymal and interstitial disease and for carrying out a precise study of the airway. The typical findings of CPL on HRCT are thickening of the interstitium, with patchy ground-glass opacities. However, HRCT must be associated with clinical findings and cannot be used as the sole diagnostic mode because the image findings are unspecific.

Since the patient presented here with diffuse interstitial infiltrate, persistent fever and coughing, treatment for TB was initiated, similar to the case reported by DIócENEs et al. [5], given the great prevalence of TB in Brazil.

Treatment of diffuse isolated CPL is palliative to prevent lymph discharge into serous cavities. Initially, the patient received medium-chain triglycerides and then parenteral nutrition with lipids, but chylothorax re-accumulated. Occurrence of refractory chylothorax is common in patients with congenital lymphatic malformations [8]. In this case, the chylothorax may have been precipitated by lung biopsy. The patient received chemical pleurodesis because consenvative treatment failed and surgical intervention was required. The most common methods are pleurectomy and chemical pleurodesis with or without thoracic duct ligation [8].

In this case, the minor alterations in the blood examination did not justify the patient's death from massive bleeding. She was being followed by a haematologist because it is likely that another disease was involved. However, she died during the investigation and no autopsy was performed. Due to the small number of reported cases, it would be premature to reach any conclusive prognosis. Of the four reported cases, including this one, three resulted in death, and the outcome after treatment was not reported in one. In contrast, BARKER et al. [9] have suggested that the prognosis of CPL with post-neonatal onset is excellent. This divergence may show that the clinical behaviour and prognosis of CPL depend on the extent of pulmonary involvement of the lymphatic dilatations and/or to other still unknown factors.

\section{Acknowledgement}

The authors would like to thank S. Xavier, from Instituto de Puericultura e Pediatria Martagão Gesteira, for the contribution to this patient's diagnosis and care.

\section{References}

1. Murray JF, Nadel JA, eds. Textbook of Respiratory Medicine. Philadelphia, W.B. Saunders, 2000; pp. 483.

2. Gardner TW, Domm AC, Brock CE, Pruitt AW. Congenital pulmonary lymphangiectasis. Clin Pediatr (Phila) 1983; 22: 75-78.

3. Brown MD, Reidbord HE. Congenital pulmonary lymphangiectasis. Am J Dis Child 1967; 114: 654-657.

4. Noonan JA, Walters LR, Reeves JT. Congenital pulmonary lymphangiectasis. Am J Dis Child 1970; 120: $314-319$.

5. Diógenes MS, de Carvalho VB, Rozov T, et al. Pericardial effusion due to pulmonary lymphangiectasis. Arq Bras Cardiol 1988; 51: 185-192.

6. Toltzis RJ, Rosenthal A, Fellows K, Castaneda AR, Nadas AS. Chylous reflux syndrome involving the pericardium and lung. Chest 1978; 74: 457-458.

7. Kelso JM, Kerr DJ, Lie JT, Sachs MI, O'Connell ER. Unusual diffuse pulmonary lymphatic proliferation in a young boy. Chest 1991; 100: 556-560.

8. Beghetti M, La Scala G, Belli D, Bugmann P, Kalangos A, Le Coultre C. Etiology and management of pediatric chylothorax. J Pediatr 2000; 136: 653-658.

9. Barker PM, Esther CR Jr, Fordham LA, Maygarden SJ, Funkhouser WK. Primary pulmonary lymphangiectasia in infancy and childhood. Eur Respir J 2004; 24: 413-419. 